\title{
CARACTERIZACIÓN DE LIDOCAÍNA Y ARTICAÍNA MEDIANTE TÉCNICA TRONCULAR EN EXODONCIAS DE TERCEROS MOLARES INFERIORES INCLUIDOS
}

\section{CHARACTERIZATION OF LIDOCAINE AND ARTICAINE THROUGH TRONCULAR TECHNIQUE IN EXODONS OF LOWER THIRD MOLARS}

\section{Baeza Solange ${ }^{1}$, Leiton Emilio², Zárate Nicole ${ }^{3}$}

\section{RESUMEN}

Objetivo: Caracterizar los efectos de tiempo de latencia, tiempo de profundidad y tiempo de duración total de articaína al $4 \%$ y lidocaína $2 \%$ ambas con epinefrina $1: 100.000$, mediante técnica troncular para la exodoncia con odontosección de terceros molares inferiores incluidos.

Materiales y Métodos: Se realizó un estudio descriptivo de tipo de serie de casos transversal seleccionada por un muestreo no probabilístico por conveniencia de 27 sujetos, con terceros molares inferiores incluidos con necesidad de odontosección donde durante el procedimiento hayan utilizado Articaína 4\% o Lidocaína al $2 \%$, ambas con epinefrina 1:100.000. Se recopilaron datos en relación al procedimiento.

Resultados: El tiempo total de duración del efecto anestésico fue mayor con el uso articaína al $4 \%$ versus lidocaína $2 \%$, siendo esto estadísticamente significativo, no así en el tiempo de latencia y el tiempo de profundidad anestésica que no presentaron significancia estadística.

Conclusión: La articaína al 4\% presenta el mejor tiempo de duración total del efecto anestésico, por lo que sería preferente para procedimientos de larga duración, permitiendo mayor comodidad post-operatoria.
1. Universidad Andrés Bello, Escuela de Odontología, Viña del Mar, Chile.

2. Atención privada odontológica, Chile.

Correspondencia:

Solange Baeza

Correo electrónico:

Solange9026@yahoo.com

\section{PALABRAS CLAVES:}

Lidocaína; articaína; anestesia local; tercer molar; exodoncia.

\section{KEYWORDS:}

Local anesthesia; lidocaine; carticaine; third molar; exodontics. 


\section{ABSTRACT}

Objective: To characterize the latency time, depth time and total duration time of $4 \%$ articaine and 2\% lidocaine- both with 1:100,000 epinephrine - during the extraction through odontosection of impacted mandibular third molars by means of a truncal anesthesia technique.

Materials and Methods: A cross-sectional descriptive study of case series selected by a non-probabilistic sampling was conducted on 27 subjects who underwent extraction through odontosection of impacted mandibular third molars using either $4 \%$ articaine or $2 \%$ lidocaine- both with 1:100,000 epinephrine. A procedure related data collection ensued.

Results: Total duration time of anesthetic effect showed a statistical significant longer effect when using $4 \%$ articaine in comparison with $2 \%$ lidocaine. Whereas, neither latency time nor anesthetic depth time showed any statistical significant difference.

Conclusion: $4 \%$ articaine provides a greater total duration time of anesthetic effect, so it should be preferred for long-term procedures, allowing greater post-operative comfort.

\section{INTRODUCCIÓN}

Para realizar la mayor parte de los tratamientos odontológicos, se hace necesario lograr un óptimo control del dolor, siendo los anestésicos locales los fármacos más utilizados para esto. ${ }^{1}$ Entre los anestésicos locales con uso autorizado en Chile se encuentran la lidocaína y la articaína. $^{2}$

Diversos estudios han sugerido que la articaína al 4\% muestra mejores características clínicas que la lidocaína al $2 \%$, especialmente en cuanto al tiempo en lograr el efecto anestésico y duración total del efecto. Según el metaanálisis cualitativo de Katyal V. que incluye 10 estudios que comparan articaína al $4 \%$ y lidocaína al $2 \%$ ambas con epinefrina 1:100.000, donde se informa de un rendimiento superior de la articaína. Esto podría mejorar la comodidad del paciente durante el intra y postoperatorio, lo cual podría suponer una importante ventaja en torno a la planificación y atención profesional. ${ }^{3}$

Los procedimientos quirúrgicos como la exodoncia de terceros molares incluidos requieren que los fármacos utilizados como anestésicos locales logren un efecto anestésico que sea de la mayor duración posible (duración total del efecto anestésico) y aseguren un bloqueo profundo en un menor tiempo posible (profundidad anestésica) de los troncos nerviosos y/o ramas terminales involucradas durante el acto quirúrgico. ${ }^{1}$

La superioridad de la articaína sobre la lidocaína presente en la literatura se ha dado únicamente al compararlas a través de una técnica de inyección infiltrativa. $^{3}$ No se han encontrado resultados estadísticamente significativos sobre la superioridad de alguno de estos anestésicos administrados a través de un bloqueo troncular en exodoncias de terceros molares incluidos que requieran odontosección.

Por lo que se busca en este estudio caracterizar los efectos de tiempo de latencia, tiempo de profundidad y tiempo de duración total de los anestésicos locales más utilizados en Chile, mediante una técnica troncular para la exodoncia con odontosección de terceros molares inferiores incluidos.

\section{MATERIAL Y MÉTODOS}

Estudio descriptivo del tipo serie de casos transversal. En el que se recolectaron los datos de pacientes sometidos a cirugía para la exodoncia de terceros molares inferiores incluidos atendidos en el pabellón de cirugía de la Facultad de Odontología de la Universidad de Valparaíso, desde el mes de agosto del 2017 hasta el mes octubre de 2018. La base de datos fue obtenida a partir de la ficha clínica de cada paciente, con previa autorización del servicio, en esta no se utilizaron datos sensibles de los pacientes y en consecuencia no fue necesario un consentimiento informado específico para el estudio. Esta base de datos es anónima e incluyó sólo a los pacientes con terceros molares inferiores incluidos que se les haya realizado exodoncias con la técnica quirúrgica de odontosección y que durante el procedimiento hayan utilizado Articaína $4 \%$ o Lidocaína al 2\%, ambas con epinefrina 1:100.000. Fueron excluidos de este 
estudio aquellos pacientes que presentaron datos incompletos dentro de la ficha. Para la selección de los pacientes se llevó a cabo un tipo de muestreo no probabilístico por conveniencia con un tamaño muestral infinito. La recopilación de los datos incluyó a las variables: tipo de fármaco anestésico utilizado, cantidad de anestésico, tiempo de latencia, tiempo de profundidad anestésica y duración del efecto anestésico. La cantidad de anestésico fue medida en número de tubos anestésicos utilizados, el tiempo de latencia se define como el tiempo considerado desde el inicio de la inyección anestésica hasta el primer efecto anestésico en los tejidos relatado por el paciente como adormecimiento de una zona en particular. Tiempo de profundidad anestésica corresponde al tiempo en que se demora desde la punción hasta el bloqueo del nervio alveolar inferior (NAI), se comprueba con punción en fondo de vestíbulo de canino inferior del lado anestesiado. Tiempo total de efecto anestésico es el tiempo considerado desde el inicio del efecto anestésico hasta el primer síntoma de dolor o molestia informado por el paciente posterior a la cirugía, lo cual fue acordado previamente con los participantes.

Los datos estadísticos se expresaron como: promedio y desviación estándar. Además, se utilizó la prueba t test de dos muestras con varianzas iguales para determinar si existía diferencia estadísticamente significativa entre la lidocaína y la articaína, comparándolas a través de las variables mencionadas anteriormente. Los análisis estadísticos y los gráficos fueron desarrollados en el software estadístico Stata 11.2.

\section{RESULTADOS}

Durante el periodo del estudio se seleccionaron un total de 27 pacientes que cumplían con los criterios de inclusión, la cantidad de anestésico utilizada para cada paciente fue de 1 tubo. Obteniendo en el análisis de las medidas de tendencia central de las variables, un tiempo de latencia para la articaína un promedio de 3.83 min $+/-1.33$ y para la lidocaína $4.18 \mathrm{~min}$ promedio $+/-1.84$, un tiempo de profundidad anestésica para la articaína un promedio de 6.12 min $+/-1.57$ y para la lidocaína un promedio de $6.33 \mathrm{~min}+/-2.42$ y un tiempo de duración total anestésico, la articaína con un promedio de $332.25 \mathrm{~min}+/-53.15$ y para la lidocaína promedio de $257.82 \mathrm{~min}+/-95.35$. (Tabla I)

$\mathrm{Al}$ realizar el análisis estadístico con la prueba $\mathrm{t}$ test de dos muestras con varianzas iguales para determinar si existieron diferencias entre las anestesias utilizadas en relación con el tiempo de latencia, se obtuvo un $\mathrm{p}$ valor de 0.5663 y el tiempo de profundidad, se obtuvo un $\mathrm{p}$ valor de 0.7925 , por lo que para estas variables no existieron diferencias estadísticamente significativas entre lidocaína y articaína.

Para la variable tiempo total del efecto anestésico se obtuvo un p valor de 0.0077 (figura 1), por lo

Tabla I: Promedio de los tiempos de latencia, profundidad anestésica y efecto anestésico según anestesia

\begin{tabular}{|c|c|c|c|c|}
\hline \multicolumn{2}{|c|}{ Anestesia } & $\begin{array}{c}\text { Tiempo } \\
\text { de } \\
\text { latencia }\end{array}$ & $\begin{array}{c}\text { Tiempo de } \\
\text { profundidad } \\
\text { anestésica }\end{array}$ & $\begin{array}{c}\text { Tiempo } \\
\text { total del } \\
\text { efecto } \\
\text { anestésico }\end{array}$ \\
\hline \multirow{2}{*}{ Articaína } & Promedio & 3.83 & 6.12 & 332.25 \\
\cline { 2 - 5 } & $\begin{array}{c}\text { Desviación } \\
\text { estándar }\end{array}$ & 1.33 & 1.57 & 53.15 \\
\hline \multirow{2}{*}{ Lidocaína } & Promedio & 4.18 & 6.33 & 257.82 \\
\cline { 2 - 5 } & $\begin{array}{c}\text { Desviación } \\
\text { estándar }\end{array}$ & 1.84 & 2.42 & 95.35 \\
\hline \multicolumn{2}{|c|}{ T-Test p-valor } & 0.5663 & 0.7925 & 0.0077 \\
\hline
\end{tabular}

Figura 1: Total del efecto anestésico según anestesia

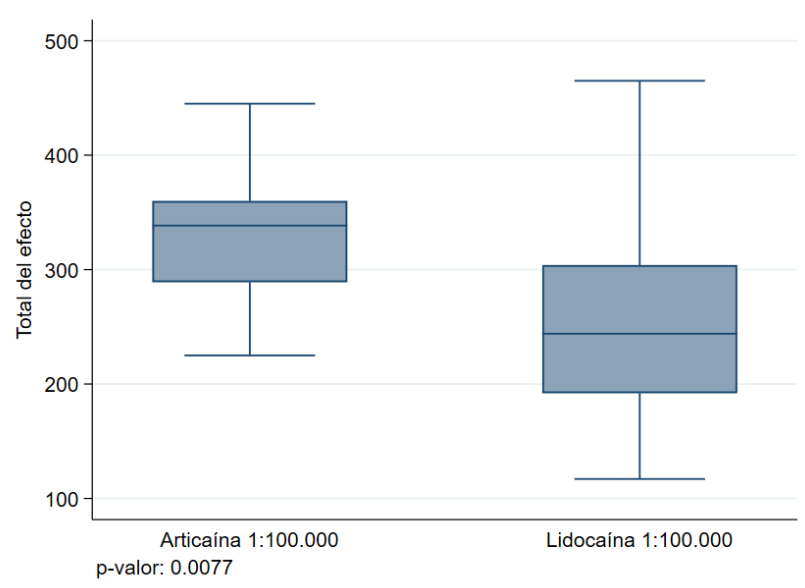


que existieron diferencias estadísticamente significativas entre los anestésicos. Además, para esta misma variable, se observó que la lidocaína presenta una mayor variabilidad de los datos, obteniendo valores extremos de 465 minutos como máxima duración y 117 minutos como mínimo, en comparación con la articaína que obtuvo valores extremos de 445 minutos y 225 minutos.

\section{DISCUSIÓN}

El principal hallazgo de este estudio es que existe diferencias estadísticamente significativas en el tiempo total del efecto anestésico de la lidocaína y la articaína, siendo la articaína al $4 \%$ el anestésico que presenta el mejor resultado con un tiempo de duración total del efecto anestésico mayor a la lidocaína al 2\%. La mayor duración total del efecto podría explicarse según lo informado por Courtney et al que dice que el grado de unión de las moléculas anestésicas a la membrana del nervio dicta la duración del efecto anestésico, por lo tanto, con una unión más potente, el anestésico es liberado más lentamente desde los sitios receptores en los canales de sodio, lo que conlleva a una duración del efecto anestésico prolongada ${ }^{4}$, lo que realiza de mejor forma la articaína que la lidocaína.

En cuanto al tiempo de latencia y profundidad anestésica existen diferencias al comparar ambos fármacos, pero estas diferencias no fueron estadísticamente significativas. A pesar de esto Bartlett $G$ y Mansoor J señalan que la articaína tiene un tiempo de latencia $y$ profundidad anestésica más corto, además de una mayor tasa de éxito. ${ }^{5}$ Lo anterior se explica porque la articaína es derivada del tiofeno en lugar del benceno, como la lidocaína, lo cual le otorga una mayor solubilidad en los lípidos, que aumenta la difusión del anestésico a través de la vaina nerviosa. ${ }^{6}$

En la revisión de la evidencia actual, no se encuentran estudios en los cuales se aplique la misma metodología aplicada en esta investigación. Sin embargo, se consideran los resultados descriptivos de diversos ensayos clínicos y metaanálisis. Es así como en un ensayo clínico realizado por Sierra-Rebolledo et al. donde se evaluaron las mismas soluciones anestésicas en exodoncia de terceros molares inferiores impactados y con una tamaño muestral similar, se observó una duración más prolongada de la anestesia con Articaína ${ }^{7}$, lo que concuerda con el principal a hallazgo del presente estudio, por lo que a la luz de estos resultados frente a procedimientos de mayor duración y complejidad sería aconsejable utilizar articaína al 4\%, así como también para mayor comodidad del paciente en el post operatorio.

Con relación al período de latencia y la duración total del efecto anestésico, un estudio realizado por Costa et al. en el año 2005, determinó un inicio más corto del efecto anestésico en el uso de Articaína en comparación con la Lidocaína, así como un mayor tiempo total del efecto anestésico de la articaína. ${ }^{8} \mathrm{Si}$ bien en cuanto al tiempo total de efecto anestésico hay concordancia con este estudio, el tiempo de inicio del efecto anestésico difiere con nuestros resultados, lo que podría deberse a la diferente metodología utilizada, donde la anestesia se aplica mediante técnica infiltrativa en el maxilar superior, que es un hueso más permeable y menos denso que la mandíbula, siendo esta última el campo de trabajo en el presente estudio mediante la aplicación de una técnica troncular.

Yapp et al., en su estudio estableció que la Articaína tiene propiedades clínicas satisfactorias en comparación a la Lidocaína y un tiempo de inicio variable con poca predictibilidad para la anestesia profunda. ${ }^{9}$ Este estudio es comparable ya que comparte el procedimiento clínico y la aplicación de ambos anestésicos, pero difiere en cuanto a la técnica de inyección, lo cual explicaría la poca predictibilidad en la profundidad anestésica, ya que en una técnica infiltrativa, el sitio anatómico infiltrado se encuentra más lejos del tronco nervioso, considerando la anestesia de este como la mayor profundidad anestésica conseguida. Es por lo anterior, que nuestro artículo considera la inyección de forma troncular para las exodoncias de terceros molares que requieran odontosección con el fin de identificar de mejor manera la profundidad anestésica y el tiempo en conseguirla. Sin embargo, la metodología aplicada de este estudio no permite la extrapolación de los resultados y, por lo tanto, los resultados del estudio de Yapp et al. ${ }^{9}$ serían más 
concluyentes, aunque se debería considerar la perspectiva investigativa aplicada en el presente estudio.

Dentro de las principales limitaciones que tuvo este estudio, es la metodología utilizada,

ya que al momento de medir la variable "tiempo de duración total del efecto anestésico" definida como la resta entre la hora de punción y la hora en la que el paciente relata el cese del efecto anestésico, donde la determinación depende de la sensación y relato de manera oportuna por parte del paciente hacia el operador, pudiendo ocurrir un error sistemático de memoria si existe alguna incoordinación en la comunicación entre el operador y el paciente, o bien, si alguno de los pacientes olvida informar el momento exacto del cese del efecto anestésico, de todas formas es preciso señalar que al momento de solicitarle al paciente su cooperación, este se compromete a informar de forma fidedigna o bien informar su olvido, por lo tanto, estos casos son eliminados de la base de datos. Para estudios posteriores se sugiere que exista algún método de evaluación y comprobación objetiva para medir esta variable o que se realice una calibración adecuada para determinarla.

Otra de las limitaciones presentes en este estudio alude a la muestra, ya que está no fue seleccionada de manera aleatoria y su tamaño no es representativo, pero se debe recalcar que el estudio se realiza en base a una situación clínica real. Para lo anterior y sumado a la metodología, se sugiere continuar esta línea investigativa aplicando una metodología experimental y con un muestreo aleatorizado, con el fin de extrapolar los datos y que a su vez estos sean significativos para contribuir con la evidencia.

\section{CONCLUSIÓN}

El tiempo total de duración del efecto anestésico es mayor con el uso articaína al $4 \%$ respecto a la lidocaína 2\% ambas con epinefrina 1:100.000, con una significancia estadística, no así en el tiempo de latencia y el tiempo de profundidad, los cuales fueron levemente menores con el uso de Articaína, pero sin llegar a tener significancia estadística. A pesar de que no todos los resultados del estudio son estadísticamente significativos, estos son concordantes con la evidencia actual presente en la literatura.

La Articaína sería preferente para procedimientos clínicos de larga duración y permitiría una mayor comodidad del paciente post operatorio.

\section{REFERENCIAS}

[1] Brandt R, Anderson P, McDonald N, Sohn W, Peters M. The pulpal anesthetic efficacy of articaine versus lidocaine in dentistry. J Am Dent Assoc. 2011;142(5):493-504. doi: 10.14219/jada.archive.2011.0219 1 y 4

[2] DS $\mathrm{N}^{\circ} 435$ 1981, Aprueba reglamento del sistema nacional de control de productos farmacéuticos, alimentos de uso médico y cosméticos, Ministerio de Salud, Gobierno de Chile

[3] Katyal V. The efficacy and safety of articaine versus lignocaine in dental treatments: A metaanalysis. J Dent. 2010;38(4):307-317. doi: 10.1016/j.jdent.2009.12.003

[4] Courtney KR, Kendig JJ, Cohen EN: The rates of interaction of local anesthetics with sodium channels in nerve. J Pharmacol Exp Ther 207:594, 1978

[5] Bartlett G, Mansoor J: Articaine buccal infiltration vs lidocaine inferior dental blockA review of the literature. Br Dent J 220:117, 2016

[6] Zhang A, Tang H, Liu S, Ma C, Ma S, Zhao H. Anesthetic Efficiency of Articaine Versus Lidocaine in the Extraction of Lower Third Molars: A Meta-Analysis and Systematic Review. J Oral Maxillofac Surg. 2019;77(1):18-28. doi: 10.1016/j.joms.2018.08.020

[7] Sierra A, Delgado E, Berini L, Gay C. Comparative study of the anesthetic efficacy of $4 \%$ articaine versus $2 \%$ lidocaine in inferior alveolar nerve block during surgical extraction of impacted lower third molars. Med Oral Patol Oral Cir Bucal. 2007; 12: E139-44.

[8] Costa G., Tortamano G, Rocha R. Onset and duration periods of articaine and lidocaine on maxillary infiltration. Quintessence Int. 2005 Mar;36(3):197- 201.

[9] Yapp K, Hopcraft M, Parashos P. Articaine: a review of the literature. $\mathrm{Br}$ Dent $\mathrm{J}$. 2011;210(7):323-329. 


\section{CÓMO CITAR ESTE ARTÍCULO}

Baeza S, Leiton E, Zárate N. Caracterización de lidocaína y articaína mediante técnica troncular en exodoncias de terceros molares inferiores incluidos. Appli. Sci. Dent. 2020; 1(1): 34-39 DOI: 10.22370/asd.2020.1.1.2111 